\title{
Structural Effects of Small Molecules on Phospholipid Bilayers Investigated by Molecular Simulations
}

\author{
Bryan W. Lee and Roland Faller* \\ Department of Chemical Engineering and Materials Science, \\ University of California-Davis, One Shields Ave, Davis, CA 95616, USA \\ Amadeu K. Sum \\ Department of Chemical Engineering, Virginia Polytechnic Institute and State University, \\ 142A Randolph Hall, Blacksburg, VA 24061, USA \\ Ilpo Vattulainen \\ Laboratory of Physics and Helsinki Institute of Physics, \\ Helsinki University of Technology, P. O. Box 1100, FIN-02015 HUT, Finland \\ Michael Patra and Mikko Karttunen \\ Biophysics and Statistical Mechanics Group, Laboratory of Computational Engineering, \\ Helsinki University of Technology, P. O. Box 9203, FIN-02015 HUT, Finland
}

\begin{abstract}
We summarize and compare recent Molecular Dynamics simulations on the interactions of dipalmitoylphosphatidylcholine (DPPC) bilayers in the liquid crystalline phase with a number of small molecules including trehalose, a disaccharide of glucose, alcohols, and dimethylsulfoxide (DMSO). The sugar molecules tend to stabilize the structure of the bilayer as they bridge adjacent lipid headgroups. They do not strongly change the structure of the bilayer. Alcohols and DMSO destabilize the bilayer as they increase its area per molecule in the bilayer plane and decrease the order parameter. Alcohols have a stronger detrimental effect than DMSO. The observables which we compare are the area per molecule in the plane of the bilayer, the membrane thickness, and the NMR order parameter of DPPC hydrocarbon tails. The area per molecule and the order parameter are very well correlated whereas the bilayer thickness is not necessarily correlated with them.
\end{abstract}

Keywords: Lipid membranes; structure and physical properties; theory and modeling; molecular simulation

\section{INTRODUCTION}

Phospholipid bilayers have been the focus of research for a long time due to their natural occurrence in cellular and intracellular membranes. Detailed computer simulations of phospholipid monolayers and bilayers have achieved

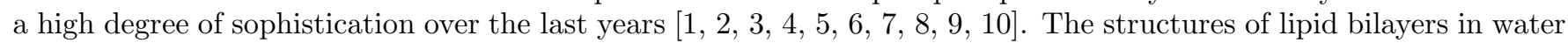
have been determined for a variety of phospholipids [4, 6, 11, 12] and the understanding of their structural features has increased significantly. More recently computational studies of mixtures of various phospholipids [12, 13, 14, 15. and of phospholipids with cholesterol [16, 17, 18, 19] have also been reported. However, the question how such lipid bilayer membranes interact with small molecules has to date not gained very much attention except for a few initial studies on alcohols [20, 21, 22], sugars 23, 24], and dimethylsulfoxide (DMSO) 25, 26]. The influence of lipophilic polymers on lipid bilayers has also been studied [27].

If we want to understand the interactions of cells with their environment, we first have to understand the interaction of cell membranes - or phospholipid bilayers as model systems - with molecules typical in cell environments. Cell membranes are the first part of the cell to come into contact with any nutrient, pathogen, or other molecule in the environment. So the understanding of membrane interaction with small molecules is of tremendous biological importance.

In this contribution we compare the structural influences of different small molecules on model lipid membranes. We are focusing on molecules with high biological relevance. These include trehalose, alcohols and dimethylsulfoxide. Sugar molecules are obviously nutrients to living organisms and some sugars are also known to be cryo-protectants 28 , 29, 30]. In particular, trehalose, which is a disaccharide of glucose has been found to be very effective in this respect. It has been pointed out that trehalose is able to form only one internal hydrogen bond in contrast to, e.g., sucrose which forms two [31]. This leaves trehalose more susceptible to hydrogen bonding with lipids. Recently, it has been shown that the molecular mechanism underlying this cryo-protective effect is the hydrogen bonding pattern of the

\footnotetext{
* to whom correspondence should be addressed at rfaller@ucdavis.edu
} 


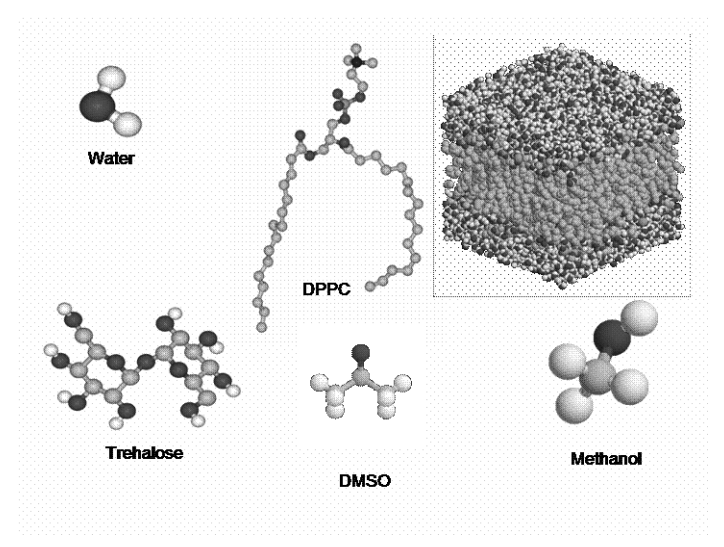

FIG. 1: The various molecules used in our simulation and a typical bilayer configuration.

trehalose molecules to the bilayer headgroups [23]. The sugar can "substitute" some of the hydrogen bonds normally provided by water and by that stabilize the fragile bilayer arrangement. Stabilization or destabilization here mean that the bilayer is able to withstand harsher or less harsh conditions in presence of small molecules compared to the pure bilayer in water. Trehalose is experimentally known to prevent the lipid from undergoing a phase transition under cooling, i.e., it shifts the main phase transition temperature significantly [32].

DMSO is known to have some cryo-protective properties as well 33], in addition to its anti-inflammatory, analgesic, muscle relaxant, mast cell stimulation and collagen dissolution properties [34]. It has been reported that DMSO strongly changes the overall bilayer structure by penetrating into the hydrocarbon layer to a much higher degree than sugar or alcohol molecules [25].

It has been observed experimentally that alcohols have a destabilizing effect on model membranes [35, 36]. It has also been observed that upon addition of alcohols, the lipid bilayer becomes thinner and the area per molecule increases. A related industrial question is the problem known as stuck fermentation in the wine industry [37, 38]. A stuck fermentation means that the yeast cells do not transform all the available sugar into alcohol but stop at an incomplete stage. It has been proposed that the underlying mechanism is an alcohol-triggered structural transition in the membrane which forces trans-membrane proteins to change conformation rendering them dysfunctional [37]. To date, there is no predictive means to detect stuck fermentations. The first approach to understand the mechanisms leading to stuck fermentation is therefore to study the influence of alcohols on lipid bilayers as the direct effect on the proteins is expected to be only secondary.

\section{SIMULATION DETAILS}

In this contribution we focus on a comparison between small molecules and their effect on the bilayer structure. The details of the simulation models are presented elsewhere 21, 23, 25]. Let us summarize the main characteristics. All simulations contain fully hydrated dipalmtoylphosphatidylcholine (DPPC) bilayers with 128 molecules, i.e., 64 per leaflet (DPPC is one of the most abundant phospholipids in animal cell membranes). Our simulations are in atomistic detail except for the hydrogen atoms bonded in methyl(ene) groups. These groups are collapsed into a united atom description centered on the respective carbon. There are at least 3655 water molecules in the system (8958 water molecules for some systems with alcohol). All simulations were performed at $325 \mathrm{~K}$, a temperature at which DPPC is in the biologically most relevant fluid phase. Simulations of the pure bilayer, as well as bilayers with up to $3 \mathrm{wt} \%$ methanol or ethanol, (lipid free basis, as we do not take the lipids into account for concentration calculations) have been performed. Note that a weight concentration of $1.7 \mathrm{wt} \%$ methanol is the same molar concentration as $2.5 \mathrm{wt} \%$ ethanol. In the case of trehalose the concentrations considered were up to $3 \mathrm{wt} \%$, simulations at higher concentrations and different temperatures have been reported elsewhere [23, 24]. The DMSO systems contained 18.6 wt\% DMSO. Simulations were performed under constant temperature and constant pressure conditions using the Berendsen weakcoupling scheme [39]. Simulations used a time-step of 1-2 fs. Figure 1shows all the used molecules as well as a typical bilayer configuration.

It is worth noting that the exact simulation conditions can have a significant effect on the structural properties. For example, changing the cutoff for the nonbonded interactions can change the area per molecule by as much as $10 \%$ [8]. Thus all direct comparisons have been performed under identical conditions with a cutoff of the LennardJones interaction of $0.9 \mathrm{~nm}$ or $1.0 \mathrm{~nm}$, respectively. The simulation models of the lipid alkyl tails used either the 
NERD [40, 41, 42] or the GROMOS force-field [43]; no significant differences were observed between these two models based on simulations of the pure bilayer system [8, 23]. This is not surprising as the two force-fields differ only slightly in their treatment of the hydrocarbon tails. The parameters for the head-groups are identical. However, if we compare our areas per molecule to the data of Pereira et al. 24] who used a different model, we see a difference of about $10 \%$ for the pure DPPC bilayer and for the systems with trehalose. The models for the alcohols use either the GROMOS forcefield [43] or the model suggested by Müller-Plathe [44], small differences in the density profiles were observed. The other observables discussed here were in all compared cases the same, a detailed comparison will be published elsewhere [45]. Trehalose was modeled using the OPLS model [46], DMSO using the revised model by Bordat et al. 47] and water using the SPC/E model [48].

Electrostatic interactions have been considered using the particle mesh Ewald technique [49]. A few simulations have been performed using a reaction field technique [50]. We compared the results from the simulations using the reaction field technique to the PME data for a representative subgroup and found no significant differences. Simulations did not contain any salt or other electrolytes.

We focus on three key observables to characterize the structure of the bilayer systems in contact with small molecules. These are the area per molecule, the membrane thickness and the alkyl tail order parameter. The area per molecule is simply defined as the surface area in the $x y$ plane (the membrane normal is the $z$ direction) divided by the number of molecules per leaflet. The membrane thickness is defined as the distance between the peaks of highest density in a mass density profile, which roughly corresponds to the distance between the planes of the phosphorus atoms.

The order parameter is defined in reference to NMR experiments by

$$
\begin{aligned}
-S_{C D} & =\frac{2}{3} S_{x x}+\frac{1}{3} S_{y y}, \\
S_{\alpha \beta} & =\left\langle 3 \cos \Theta_{\alpha} \cos \Theta_{\beta}-\delta_{\alpha \beta}\right\rangle, \quad \alpha, \beta=x, y, z \\
\cos \Theta_{\alpha} & =\hat{e}_{\alpha} \hat{e}_{z},
\end{aligned}
$$

where $\hat{e}_{z}$ is a unit vector in the laboratory $z$-direction and $\hat{e}_{\alpha}$ is a unit vector in the local coordinate system of the tails, which involves three connected carbons $\mathrm{C}_{i-1}, \mathrm{C}_{i}$, and $\mathrm{C}_{i+1}$ and $\vec{e}=\vec{r}_{i+1}-\vec{r}_{i-1}$. This order parameter characterizes the alignment of the hydrocarbon tails with the bilayer normal. It can be used as one of the parameters (in addition to, e.g., the area per molecule and the thickness) to characterize the thermodynamic phase of the system. All simulations in this contribution are in the biologically most relevant fluid phase. At a lower temperature the order of the tails increases and this triggers a phase transition to the gel phase.

All these observables are experimentally accessible; the order parameter and the area per molecule can be measured by NMR. The thickness can be measured experimentally by using electron density profiles from X-ray scattering [51]. However, especially under the influence of small molecules it is often inferred indirectly from the area per molecule under the assumption of volume conservation in the bilayer [35].

\section{BILAYER STRUCTURE AND INFLUENCE OF SMALL MOLECULES}

At the temperature of $325 \mathrm{~K}$ we found the area per molecule to be $0.65 \mathrm{~nm}^{2}$ for a pure DPPC bilayer which lies well within experimentally reported values of $0.55 \mathrm{~nm}^{2}$ to $0.71 \mathrm{~nm}^{2}$ [51, 52, 53, 54]. The alkyl tail order parameters for the pure DPPC system, shown in Fig. 2] are also consistent with experimental data [55] and previous simulation studies [7]. The area per molecule increases and the order parameter decreases with increasing temperature in the pure system [23]. Moreover, the order parameter decreases continuously from the head group to the end of the tails in the bilayer center. This means that the tails in the center of the bilayer are only very weakly aligned with the bilayer normal, especially at high alcohol and DMSO concentrations. It is conceivable that at higher alcohol concentrations the order may be completely lost. At very high alcohol concentrations interdigitation between opposing monolayers has been experimentally observed [56]. Here, no interdigitation was observed as we study much lower concentrations (cf. Fig. [3b). The thickness of pure bilayers shrinks as the phospholipids become less ordered. This means that in the $z$-direction - defined to be the normal to the bilayer surface - one finds a negative heat expansion coefficient. The overall heat expansion coefficient is positive as the increase in area overcompensates the loss in thickness. Figure 3 ; shows the density profile of a pure DPPC bilayer. We clearly see the self-assembly of the system into a water region with a density very close to bulk water, an interface with a high local density as the heavy phosphorus atoms are located in this area, and an aliphatic region well below water density in the center. The plane of lowest density in the middle of the bilayer where the lipid tails from opposing leaflets meet is a symmetry plane, which we define as the $z=0$ plane. The interface region contains some water and the lipid head groups, which include the choline and phosphate groups with the density increasing up to around $1400 \mathrm{~kg} / \mathrm{m}^{3}$. A small addition of $1.0 \mathrm{wt} \%$ methanol leads to an overall decrease of the bilayer density which suggests an increase in the area per molecule. This is indeed 


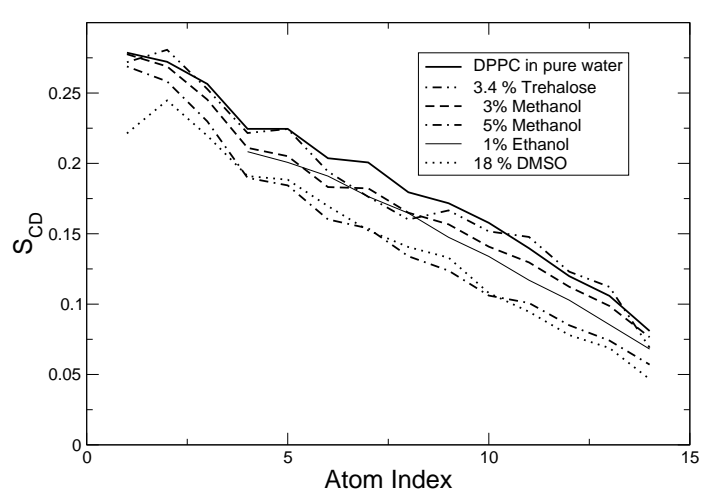

FIG. 2: Order parameter for DPPC at $325 \mathrm{~K}$ in dependence of concentration of added small molecules. We average over both tails. Note that the data for trehalose is different from the one presented in [23] as we are presenting data at a lower temperature. Carbon number 1 is closest to the headgroup and carbon number 15 is the end of the tails.

\begin{tabular}{|c|c|c|}
\hline Molecule & weight $\%$ area per molec. & {$\left[\mathrm{nm}^{2}\right]$} \\
\hline pure water & 0 & 0.65 \\
\hline \multirow[t]{3}{*}{$\mathrm{MeOH}$} & 1 & 0.69 \\
\hline & 1.7 & 0.69 \\
\hline & 3 & 0.72 \\
\hline $\mathrm{EtOH}$ & 2.5 & 0.70 \\
\hline Trehalose & 3 & 0.64 \\
\hline DMSO & 18 & 0.72 \\
\hline
\end{tabular}

TABLE I: Dependence of the area per molecule at $325 \mathrm{~K}$ under addition of different molecules. Note that the concentrations are weight $\%$ lipid free basis. The Error estimates are $\pm 0.01 \mathrm{~nm}^{2}$ unless otherwise noted.

observed (cf. Table. I), as well as an increase in the bilayer thickness, leading to an overall decrease of bilayer density. This result actually challenges the typical experimental assumption of constant bilayer density often used to calculate changes in layer thickness [35, 36]. To characterize the structure of the bilayer at least two independent measurements out of the set thickness, area, and density are necessary.

Experimentally it is found that low molecular weight alcohols increase the area per molecule and decrease the order. The experimental increase for an SOPC bilayer [36] is of the order of $\Delta A / A \approx 0.1$ at $5 \mathrm{wt} \%$ methanol. Consequently, the thickness of the membrane is inferred to be thinned out. Measurements using DPPC or other fully saturated phosphatidylcholines have not yet been reported. The only simulation study of alcohols on bilayers we are aware of [20] did not allow for changes in the box-size in the plane of the membrane as that study focused on local structural effects and dynamics, and hence it is not possible to directly compare our results to those of Feller et al. [20]. Here we find a linear dependence of the area expansion on alcohol concentration as well as an increase of the effect from methanol to ethanol which is in line with the experimentally observed increase of the area expansion with alcohol chain length [36]. A more detailed analysis of the alcohol-bilayer interaction is found in Ref. [21].

Bemporad et al. measured free energies of transition through a lipid bilayer for various small molecules [22]. Although their numbers for the permeability are an order of magnitude too large, it is clear that methanol faces a barrier which is almost as high as the one for water. It is highly unlikely for the methanols to cross the bilayer. This is very much in agreement with our density profiles as we do not find any methanol molecules inside the layer. For ethanol, however, a few molecules were found inside the layer in agreement with the old Traube rule [57] which says that interfacial tension drops by a constant factor as one adds methylene groups.

The simulations with trehalose revealed only small changes to the lipid bilayer. We found a slight increase in the order parameter with increasing sugar concentration; this effect was earlier found to be more pronounced for trehalose than for its isomer sucrose 23]. We find that trehalose stabilizes and preserves the membrane under cooling without affecting its structure. This is understandable from the point of view of cryo-preservation and also due to the fact taht sugars are an abundant nutrient of cells and membrane integrity must not be strongly affected by their presence (see ref. 23. for additional results and discussions). As seen from the density profiles in Fig. 3:, trehalose does not penetrate into the bilayer and accumulates at the headgroups. The sugar molecules hydrogen bond to the lipid headgroups, one trehalose molecule can bind to up to three lipids.

Pereira et al. performed simulations of a DPPC bilayer in water with much higher concentrations of trehalose and 

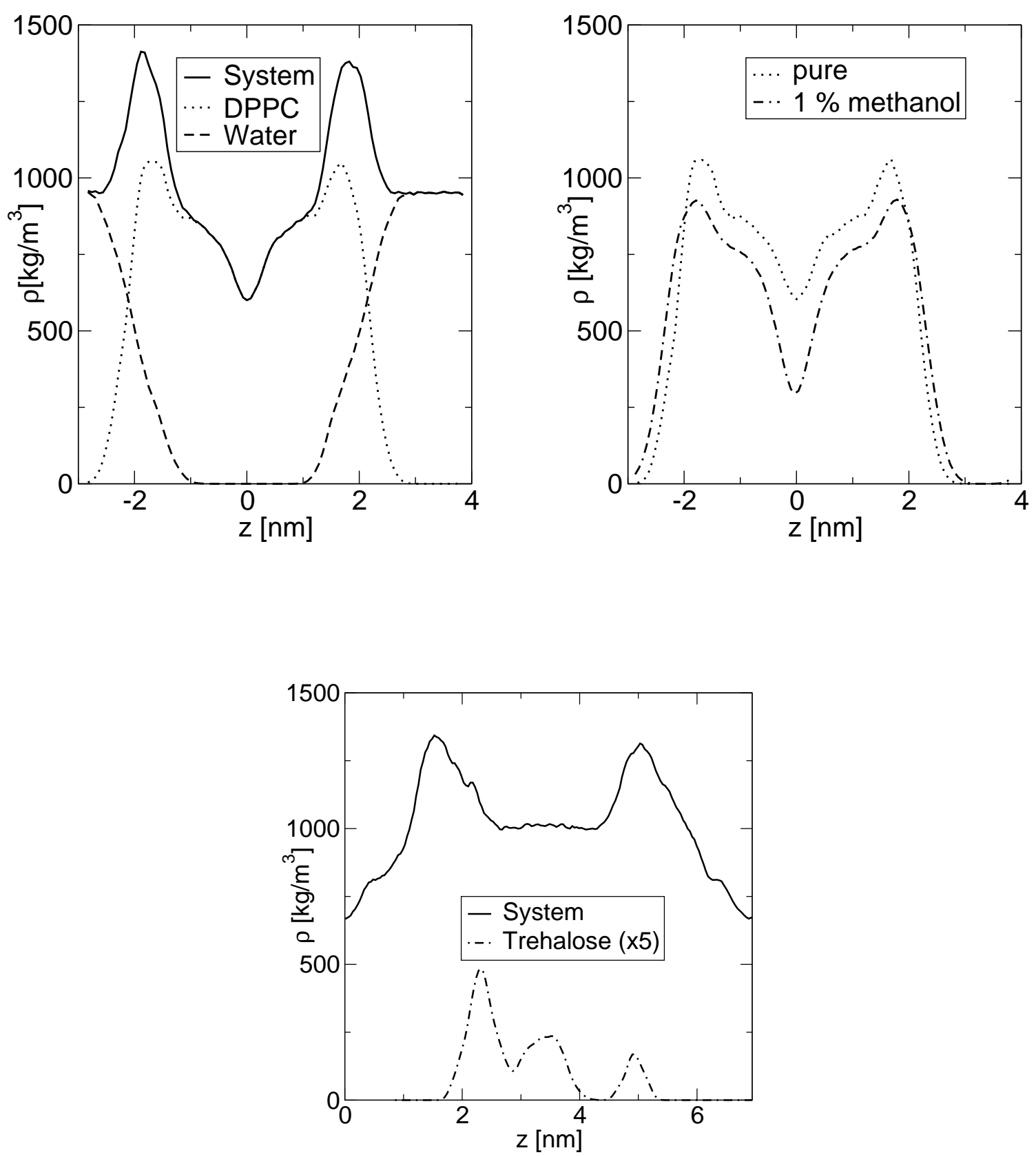

FIG. 3: Density profiles a) of a pure DPPC system and its constituents b) pure system in comparison with a system containing 1 wt $\%$ methanol (using the model from Ref. 44]. We only show the density profile of the lipids here. c) system containing 3.4 wt $\%$ trehalose, these curves are centered around the middle of the water layer $(z=3.47 \mathrm{~nm})$ for clarity. Note that the asymmetry in the trehalose distribution is a statistical effect and not a sign of insufficient equilibration.

found very similar effects 24]. The area per molecule is almost unaffected even with 128 molecules of trehalose for 128 molecules of DPPC. The same is true for the order parameter. Their simulation model is different so that the area per molecule cannot be compared directly, they get $0.58 \mathrm{~nm}^{2}$ for the pure DPPC system at $325 \mathrm{~K}$. But it is clear that the effect of trehalose is very similar in two independent studies with distinctly different models.

For the bilayer system containing DMSO, the area per molecule is increased, but not as strongly as in the presence of methanol or ethanol, and consequently we observe a decrease in the order parameter. A concentration of about $18 \mathrm{wt} \%$ DMSO is needed to get the same increase in area per molecule as with $3 \mathrm{wt} \%$ methanol. Even if the molar 
concentrations are considered for comparison, we obtain the same effect with $1.6 \mathrm{~mol} \%$ methanol versus 5 mol\% DMSO.

As described earlier, we see an increase in the thickness of the bilayer upon addition of methanol. We measured the thickness of the bilayer as the distance between the planes of highest density of the overall system. In the pure system (DPPC and water only) we obtained a layer thickness of $3.68 \mathrm{~nm}$. Addition of $1 \mathrm{wt} \%$ methanol gave $4.00 \mathrm{~nm}$, whereas $18 \mathrm{wt} \%$ DMSO lead to a decrease to $3.24 \mathrm{~nm}$. This suggests that the change in the bilayer thickness can not be directly inferred from the change in the area per molecule. Again we see that trehalose has the weakest influence with a thickness of $3.50 \mathrm{~nm}$. The errors on the thickness estimation are at most around $0.2 \mathrm{~nm}$.

\section{CONCLUSIONS}

We have found, in agreement with experimental data, that trehalose has a stabilizing effect on lipid bilayers. The bilayer structure is essentially unchanged from the structure of the pure system even if the temperature is lowered [23, 30]. However, the presence of alcohol or DMSO is contrastingly different: small concentrations of alcohol have a detrimental effect on the bilayer structure, the influence of DMSO being somewhat less pronounced. The area per molecule and bilayer thickness increase in the presence of alcohols, leading to a decrease in the overall density of the bilayer. The opposite effect is observed with DMSO, as the bilayer density is relatively unchanged, and the area per molecule increase leads to a thinning of the bilayer.

These results suggest that the area per molecule and the alkyl tail order parameter are strongly anti-correlated, that is, an increase in the order parameter is accompanied by a decrease in area per molecule and vice-versa. Similar results have been found in a recent study on chesterol phospholipid interaction [19]. This is understood as the area per molecule describes the two-dimensional packing density of the headgroups and the tails. The closer the alkyl tails are packed, the more they are ordered. It is generally accepted that the phase transition to the gel phase with a strong increase in order parameter - at lower temperatures is due to tail packing. At this moment, it is not clear if there is a correlation of the bilayer thickness to the degree different molecules penetrate the bilayer. However, even this cannot explain our observations as DMSO penetrates the bilayer better than the alcohols [20, 21, 25]. This question remains open for the moment and further investigations are needed.

In the light of our findings, that there is no direct correlation between bilayer thickness and area per molecule, we would recommend to use scattering experiments to obtain the layer thickness and not to rely on the assumption of constant bilayer volume.

We conclude that atomistic simulations are a powerful means to study the interaction of small molecules with model lipid bilayers and can give us detailed insight on the local mechanisms of interactions. In order to understand the larger scale effects, simulations with simpler models have to be applied [58, 59, 60, 61].

\section{Acknowledgments}

One of the authors (R. F.) thanks H. V. Ly, M. Longo, F. Tablin, and J. H. Crowe for interesting discussions on the experiments. We are pleased to acknowledge the support by the Academy of Finland through its Center of Excellence Program (I. V.), the European Union through the Marie Curie fellowship HPMF-CT-2002-01794 (M.P.), and the Academy of Finland Grant Nos. 00119, 54113 (M. K.), and 80246 (I. V.). We would also like to thank the Finnish IT Center for Science (CSC) and the HorseShoe (DCSC) supercluster computing facility at the University of Southern Denmark for computer resources as well as Advanced Microdevices Inc. for the donation of processors (R. F.). 


\section{List of Symbols and Abbreviations}

$\begin{array}{ll}A & \text { area per molecule in the plane of the bilayer } \\ \delta_{\alpha \beta} & \text { Kronegger Delta, } \delta=1 \text { if } \alpha=\beta \text { otherwise } 0 \\ \Delta A & \text { change in the area per molecule in the plane of the bilayer } \\ \text { DMSO } & \text { dimethylsulfoxide } \\ \text { DPPC } & \text { dipalmitoyl-phosphatidylcholine } \\ \text { GROMOS } & \text { Groningen Molecular Simulation } \\ \text { NERD } & \text { Nath, Escobedo, de Pablo, revised force-field } \\ \text { NMR } & \text { nuclear magnetic resonance } \\ \text { PME } & \text { Particle Mesh Ewald } \\ S_{C D} & \text { NMR alkyl tail order parameter } \\ S_{x x}, S_{y y} & \text { components of the order parameter tensor } \\ \text { SOPC } & \text { stearoyl-oleoyl-phosphatidylcholine } \\ z & \text { coordinate along the bilayer normal } \\ \Theta_{\alpha} & \text { angle between unit vector } \alpha \text { and the bilayer normal }\end{array}$

References

[1] S. E. Feller et al., J Chem Phys, 103(1995) 10267-10276.

[2] D. J. Tobias et al., Curr Opin in Coll \& Int Sc, 2(1997) 15-27.

[3] S. Bandyopadhyay et al., Curr Opin in Coll \& Int Sc, 3(1998) 242-246.

[4] T. Husslein et al., J Chem Phys, 109(1998) 2826-2832.

[5] T. Rog and M. Pasenkiewicz-Gierula, Biophys J, 81(2001) 2190-2202.

[6] L. Saiz and M. L. Klein, J Chem Phys, 116(2002) 3052-3057.

[7] D. P. Tieleman et al., Biochim Biophys Acta (Rev on Biomem), 1331(1997) 235-270.

[8] M. Patra et al., Biophys J, 84(2003) 3636-3645.

[9] H. Leontiadou et al., Biophys J, 86(2004) 2156-2164.

[10] M. Patra et al., J Phys Chem B, 108(2004) 4485-4494.

[11] R. J. Mashl et al., Biophys J, 81(2001) 3005-3015.

[12] A. Gurtovenko et al., Biophys J, 86(2004) 3461-3472.

[13] S. A. Pandit et al., Biophys J, 85(2003) 3120-3131.

[14] K. Balali-Mood et al., Eur Phys J E, 12(2003) S135-S140.

[15] A. H. de Vries et al., J Phys Chem B, 108(2004) 2454-2463.

[16] A. M. Smondryev and M. L. Berkowitz, Biophys J, 78(2000) 1672-1680.

[17] K. Tu et al., Biophys J, 75(1998) 2147-2156.

[18] S. A. Pandit et al., Biophys J, 86(2004) 1345-1356.

[19] E. Falck et al., Lessons of slicing membranes: Interplay of packing, free area and lateral diffusion in phospholipid/cholesterol bilayers (2004), Biophys J in press (http://www.arxiv.org/abs/cond-mat/0402290).

[20] S. E. Feller et al., Biophys J, 82(2002) 1396-1404.

[21] M. Patra et al., Under the influence of alcohol: The effect of ethanol and methanol on lipid bilayers (2004), submitted to Biophys J.

[22] D. Bemporad et al., J Phys Chem B, 108(2004) 4875-4884.

[23] A. K. Sum et al., Biophys J, 85(2003) 2830-2844.

[24] C. S. Pereira et al., Biophys J, 86(2004) 2273-2285.

[25] A. K. Sum and J. J. de Pablo, Biophys J, 85(2003) 3636-3645.

[26] A. M. Smondryev and M. L. Berkowitz, Biophys J, 76(1999) 2472-2478.

[27] U. Jeng et al., Physica B, 336(2003) 204-210.

[28] J. H. Crowe et al., Biochem J, 242(1987) 1-10.

[29] J. H. Crowe et al., Biochim et Biophys Acta (Rev on Biomem), 947(1988) 367-384.

[30] J. H. Crowe et al., Cryobiology, 43(2001) 89-105.

[31] P. Conrad and J. J. de Pablo, J Phys Chem, 103(1999) 4049-4055.

[32] J. H. Crowe et al., Science, 223(1984) 701-703. 
[33] R. Freshney, Culture of Animal Cells: A Manual of Basic Technique, Alan R. Liss Inc, New York (1987).

[34] S. W. Jacob and R. Herschler, Cryobiology, 24(1986) 14-27.

[35] H. V. Ly et al., Langmuir, 18(2002) 9888-8995.

[36] H. V. Ly and M. L. Longo, The influence of short-chain alcohols on interfacial tension, mechanical properties, area/molecule, and permeability of fluid lipid bilayers (2004), Biophys $J$ in press.

[37] L. F. Bisson and D. E. Block, in M. Ciani (ed.), Biodiversity and Biotechnology of Wine Yeasts, Research Signpost, Kerala (2002) pp. 85-98.

[38] A. C. Cramer et al., Biotechnol. Bioeng., 77(2002) 49-60.

[39] H. J. C. Berendsen et al., J. Chem. Phys., 81(1984) 3684-3690.

[40] S. K. Nath et al., J. Chem. Phys., 108(1998) 9905-9911.

[41] S. K. Nath and J. J. de Pablo, Mol. Phys., 98(2000) 231-238.

[42] S. K. Nath et al., J. Chem. Phys., 114(2001) 3612-3616.

[43] W. F. van Gunsteren et al., Biomolecular simulation: The GROMOS manual and user guide, Vdf, Zürich (1996).

[44] F. Müller-Plathe, Mol Sim, 18(1996) 133-143.

[45] R. Faller et al., Comparison of various alcohol models and their effects in lipid bilayer simulations (2004), in preparation.

[46] W. Damm et al., J Comput Chem, 18(1997) 1955-1970.

[47] P. Bordat et al., Chem Phys Lett, 374(2003) 201-205.

[48] H. J. C. Berendsen et al., J Phys Chem, 91(1987) 6269-6271.

[49] U. Essmann and M. L. Berkowitz, Biophys J, 76(1999) 2081-2089.

[50] M. P. Allen and D. J. Tildesley, Computer Simulation of Liquids, Clarendon Press, Oxford (1987).

[51] J. F. Nagle et al., Biophys J, 70(1996) 1419-1431.

[52] J. F. Nagle, Biophys J, 64(1993) 1476-1481.

[53] J. F. Nagle and S. Tristram-Nagle, Curr Opin Struct Biol, 10(2002) 474-480.

[54] J. F. Nagle and S. Tristram-Nagle, Biochim Biophys Acta (Rev on Biomem), 1469(2000) 159-195.

[55] H. Schindler and J. Seelig, Biochemistry, 14(1975) 2283-2287.

[56] J. X. Mou et al., Biochemistry, 33(1994) 9981-9985.

[57] I. Traube, Liebigs Annalen der Chemie, 265(1891) 27-55.

[58] R. Faller and T. L. Kuhl, Soft Materials, 1(2003) 343-352.

[59] J. C. Shelley et al., J Phys Chem B, 105(2001) 4464-4470.

[60] S. J. Marrink et al., J Phys Chem B, 108(2004) 750-760.

[61] R. Faller and S.-J. Marrink, Simulation of domain formation in mixed DLPC-DSPC lipid bilayers (2004), Langmuir in press. 